\title{
Are All Units Created Equal? The Effect Of Default Units On Product Evaluations.
}

\begin{abstract}
Previous research on numerosity effects has shown that people often infer higher quantity from larger numbers (i.e. specified in smaller units). We argue that consumers have default units for many attribute levels. In three studies, we demonstrate that products described in default units generate more positive product evaluations, irrespective of the nominal value of the attribute (Study 1). This default unit effect is eliminated by a misattribution paradigm in which participants attribute the metacognitive feelings generated by default units to an irrelevant source (Study 2). Further, the default unit effect is eliminated for participants with an abstract mindset (Study 3).
\end{abstract}

\section{EXTENDED ABSTRACT}

Prior research on numerosity effects has yielded converging evidence that consumers often infer bigger quantity from bigger numbers (e.g. Pandelaere, Briers, and Lembregts 2011). For instance, an attribute difference looms larger when it is expressed in large numbers (i.e. small units) rather than small numbers (i.e. large units). However, past research seems to be based on the assumption that the units in which information is conveyed do not differ in evoked meaning. Consequently, the choice of specific unit in which attribute levels may be expressed seems arbitrary. In contrast with this assumption, we argue that for many attribute levels default units exist. For instance, consumers are probably more accustomed to see battery life expressed in days rather than in hours; days then constitutes their default unit for battery life. This raises the question whether consumers would still infer longer battery life if it is specified in hours (i.e. in larger numbers) rather than in days (i.e. in smaller numbers).

We offer converging evidence that this is not the case. In general, we propose that attributes expressed in default units typically generate more positive product evaluations due to enhanced ease of processing (i.e. fluency; Schwarz 2004), irrespective of the nominal value of the attribute.

Drawing on the stream of literature on numerical cognition, we suggest that the existence of default units is in many cases rooted in the way numbers are represented in the human brain (Cohen Kadosh, Tzelgov, and Henik 2008). Specifically, precision of number representation declines rapidly with increasing number size. Given that smaller natural numbers are represented more precisely in the human brain, we argue that units which provide information in smaller numbers are often preferred and therefore more frequently used as defaults. This notion is consistent with research examining frequency of numbers in language. This research confirms that smaller numbers are used more often than larger numbers in daily communication (Dehaene and Mehler 1992). In the case of battery length, people prefer a low score on this attribute to be expressed in minutes or hours. For a higher score, people prefer days. 
In a pilot study, we asked 50 participants to imagine a new product attribute. We told participants that this new product attribute did not yet have a scale onto which information was mapped. Participants were then asked to choose a range of numbers out of which the scale should consist. Participants could choose between three scales: the first scale contained numbers ranging from 10 to 100 , the second ranged from 0,1 to 1 and the third had a 100 to 1000 range. In line with our theorizing, $70 \%$ of the participants preferred the scale containing smaller natural numbers (i.e. 10-100).

In study 1, 74 participants were randomly assigned to one of two conditions and all were presented with an advertisement for a smartphone. The smartphone was described on one attribute: warranty. In the high numbers - non default condition, warranty was specified in weeks ( 80 weeks). Here, the attribute was specified in rather small units (i.e. in large numbers). In the low numbers - default condition, information was presented in years $(1,5$ years). In this case, warranty was presented in rather large units (i.e. in small numbers). A pilot study showed that for warranty 1,5 years was rated as more familiar than 80 weeks.

In line with our hypotheses, a smartphone described in default units generated a higher willingness to pay $\left(M_{\text {default }}=€ 161\right)$ than when it was specified in non-default units $\left(\left(M_{\text {non- }}\right.\right.$ default highnum $=€ 128 ; \mathrm{F}(1,74)=4.79, \mathrm{p}<.05)$.

In experiment 2, we wished to unravel the mechanism behind this effect. To achieve this, we utilized a misattribution paradigm (Schwarz et al. 1991). Background music was playing during the session. In the non-diagnostic music conditions, participants were informed that they were invited to evaluate a cell phone and answer some additional questions afterwards. Here, no specific information was given about the background music. In the diagnostic music conditions, we told participants that the experiment dealt with the influence of music on product evaluations. Moreover, we told them that previous research had shown that music has an influence on experienced feelings. Next, all participants were shown a cell phone for which warranty was displayed. In the default unit condition, warranty was expressed in years. In the non-default unit condition, warranty was specified in days. Note that, relative to the former condition, the face value of the latter condition was substantially higher.

In the non-diagnostic music conditions, planned contrasts showed a significant difference in attractiveness ratings between default and non-default unit conditions $(F(1,85)=$ $8.17, \mathrm{p}<.05)$. That is, the cell phone was rated more attractive when warranty was specified in years (default unit) than when it was specified in days (non-default unit) $\left(\mathrm{M}_{\text {default }}=6.28 \mathrm{vs}\right.$. $\mathbf{M}_{\text {non-default }}=4.47$ ). This pattern replicates the key finding of experiment 1 . Further, consistent with our hypothesis, in the diagnostic music conditions, this difference in attractiveness was not significant $\left(\mathrm{M}_{\text {default }}=5.30\right.$ vs. $\left.\mathrm{M}_{\text {non-default }}=5.72 ; \mathrm{F}(1,85)=.50, \mathrm{p}=.48\right)$. In these conditions, participants discounted the metacognitive cues generated by the attribute information.

In study 3, we draw on the finding that people who habitually engage in abstract thinking are less influenced by metacognitive cues (Tsai and Thomas 2011). Participants' prevailing construal style was administered by a modified version of the Behavioral Identification Form (Vallacher and Wegner 1989). Analyses revealed an interaction between attribute frame and prevailing construal style $(\mathrm{F}(1,154)=4.03, \mathrm{p}<.05)$. Participants with a prevailing concrete mindset were willing to pay more when a cell phone was described in 
default units $(\beta=1.17, \mathrm{t}(154)=2.21, \mathrm{p}<.05)$. For participants with a prevailing abstract mindset, however, no differences were found between the two attribute frames $(\mathrm{p}=.53)$.

In sum, the present work offers a more nuanced understanding of how specifying attributes in an alternative unit influences consumer's evaluations. Specifying attribute information in alternative units alters metacognitive experiences and thereby affects product evaluations.

\section{References}

Cohen Kadosh, Roi, Joseph Tzelgov, and Avishai Henik (2008), "A synesthetic walk on the mental number line: The size effect." Cognition, 106 (1), 548-57.

Dehaene, Stanislas and Jacques Mehler (1992), "Cross-linguistic regularities in the frequency of number words," Cognition, 43 (1), 1-29.

Pandelaere, Mario, Barbara Briers, and Christophe Lembregts (2011), "How to Make a 29\% Increase Look Bigger: Unit Effect in Option Comparisons," Journal of Consumer Research, 38(2), 308-22.

Schwarz, Norbert, Fritz Strack, Gisela Klumpp, Helga Rittenauer-schatka, and Annette Simons (1991),"Ease of Retrieval as Information: Another Look at the Availability Heuristic,” Journal of Personality and Social Psychology, 61 (2), 195-202.

Tsai, Claire I. and Manoj Thomas (2011), "When Does Feeling of Fluency Matter?: How Abstract and Concrete Thinking Influence Fluency Effects," Psychological Science, 22 (3), 348-54.

Vallacher, Robin R. and Daniel M. Wegner (1989), "Levels of personal agency: Individual variation in action identification," Journal of Personality and Social Psychology, 57 (4) 660-71. 